\title{
Volumen sanguíneo intratorácico versus presión arterial de oclusión pulmonar como estimadores de precarga cardíaca en pacientes críticos
}

\author{
Vinko Tomicic $F^{1}$, Jerónimo $\mathbf{G}$ raf $S^{1 a}$, G hislaine Echevarría $0^{2 b}$, \\ Mauricio Espinoza $R^{1}$, Juan Abarca $Z^{1}$, José Miguel Montes $S^{1}$, \\ Javier Torres $\mathrm{M}^{1}, \mathrm{G}$ astón $\mathrm{N}$ úñez $\mathrm{U}^{1}$, Julia G uerrero $\mathrm{P}^{1}$, \\ Mario Luppi ${ }^{1}{ }^{1}$, Claudio Canals $L^{1}$. \\ Intrathoracic blood volume versus pulmonary \\ artery occlusion pressure as estimators of \\ cardiac preload in critically ill patients
}

Background: Monitoring of cardiac preload by determination of pulmonary artery occlusion pressure (PAOP) has been traditionally used to guide fluid therapy to optimize cardiac output (CO). Since factors such as intrathoracic pressure and ventricular compliance may modify PAOP, volumetric estimators of preload have been developed. The PiCCO system is able to measure CO and intrathoracic blood volume (ITBV) by transpulmonary thermodilution. Aim: To compare a volumetric (ITBV) versus a pressure (PAOP) determination to accurately estimate cardiac preload in severely ill patients. Patients and Methods: From June 2001 to October 2003, 22 mechanically ventilated patients with hemodynamic instability underwent hemodynamic monitoring with pulmonary artery catheter (PAC) and PiCCO system. ITBV index (ITBVI), PAOP and CI were measured simultaneously by both methods. One hundred thirty eight deltas $(\Delta)$ were obtained from the difference of ITBVI, PAOP, CI-PAC and CI-PiCCO between 6-12 am and 6-12 pm. Linear regression analysis of $\Delta$ ITBVI versus $\Delta$ CI-PiCCO and $\Delta$ PAOP versus $\Delta$ CI-PAC were made. Results: Mean age of patients was $60.8 \pm 19.4$ years. APACHE II was $23.9 \pm 7$. Fifteen patients met criteria for acute respiratory distress syndrome (ARDS). Delta ITBVI significantly correlated with $\Delta$ CI-PiCCO ( $\mathrm{r}=0.54 ; 95 \%$ confidence interval $=0.41-0.65 ; \mathrm{p} \varangle 0.01$ ). There was no correlation between $\Delta$ PAOP and $\Delta$ CI-PAC. Conclusion: ITBVI correlated better with CI than PAOP, and therefore it seems to be a more accurate estimator of preload in unstable, mechanically ventilated patients (Rev Méd Chile 2005; 133: 625-31).

(Key Words: Blood volume; Critical illness; Pulmonary artery)

Recibido el 8 de julio, 2004. Aceptado en versión corregida 5 de abril, 2005.

${ }_{1}^{1}$ Departamento de Paciente Crítico, Clínica Alemana de Santiago. Facultad de Medicina. Clínica Alemana - Universidad del Desarrollo. Santiago de Chile. ${ }^{2}$ Departamento de Medicina, Facultad de Medicina, Universidad de Chile.

aBecario de Medicina Intensiva Adultos, Clínica Alemana - Universidad del Desarrollo.

${ }^{b}$ Becaria de Medicina Interna, Universidad de Chile.

Correspondencia a: Vinko Tomicic Flores. Av. Vitacura

5951, Santiago. Teléfono 2101600. Fax 2101068.

E-mail: vinkotomicic@udd.cl 
$E^{n}$ los pacientes críticos el principal objetivo hemodinámico es restaurar la perfusión tisular, para lo cual es necesario corregir la presión arterial y optimizar el gasto cardíaco (GC). La precarga cardíaca es la principal determinante del GC, razón por la cual la administración de fluidos es la estrategia terapéutica fundamental. Sin embargo, en el intento de alcanzar la precarga necesaria para obtener el máximo GC se puede producir sobrecarga ventricular y edema pulmonar' ${ }^{1}$.

Desde la introducción del catéter de arteria pulmonar (CAP), la determinación del GC por termodilución pulmonar (TDP) y la estimación de la precarga ventricular izquierda, a través de la presión arterial de oclusión pulmonar (PAOP), ha sido la base del monitoreo de la terapia con fluidos ${ }^{2}$. La precisión de la PAOP como indicador de precarga cardíaca ha sido cuestionada porque la medición se ve afectada por la presión intratorácica y las distensibilidades ventrícular y tóracopulmonar ${ }^{3-8}$.

El sistema PiCCO (Pulse Contour Cardiac Output), (Pulsion Medical Systems, Munich, Alemania) es un método de monitoreo hemodinámico recientemente incorporado en la práctica clínica capaz de medir el GC por termodilución transpulmonar (TDTP) y estimar la precarga por medio del volumen sanguíneo intratorácico $(\text { ITBV })^{9}$.

Existe evidencia que el ITBV es independiente de las variaciones de la presión intratorácica y distensibilidad ventricular, razón por la cual podría ser mejor estimador de precarga cardíaca que la PAOP, especialmente en pacientes que reciben ventilación mecánica con presión positiva ${ }^{10}$. Nuestro objetivo fue comparar la relación de dos estimadores de precarga (ITBV y PAOP) con el gasto cardíaco obtenido con los métodos respectivos en pacientes críticos ventilados mecánicamente.

\section{MATERIAL Y MÉTODO}

Se estudiaron, en forma prospectiva, pacientes mayores de 18 años que requirieron ventilación mecánica por más de $24 \mathrm{~h}$ y drogas vasoactivas a pesar de reanimación adecuada con volumen (PAOP $\geq 12 \mathrm{mmHg}$ ), que ingresaron a nuestra unidad entre junio 2001 y octubre 2003. Todos los pacientes se encontraban monitorizados con CAP y contaban además con un catéter venoso central para la administración de medicamentos. Se excluyeron pacientes con insuficiencia cardíaca capacidad funcional NYHA III-IV y embarazadas. La línea arterial femoral 16 Gauge (Certofix ${ }^{\circledR}$ ) Mono S 320, B Braun, Melsungen, Alemania) fue reemplazada por un catéter arterial femoral 5 French $(1,67 \mathrm{~mm})$ que se describe a continuación.

En cada paciente se efectuaron perfiles hemodinámicos sucesivos con los dos métodos a las 6 am, 12 pm, 6 pm y 12 am, según el protocolo de monitoreo hemodinámico de nuestra unidad. Adicionalmente se efectuaron determinaciones de gasto cardíaco por ambos métodos cuando la situación clínica lo ameritaba. La conducta clínica fue guiada por la información obtenida desde el $\mathrm{CAP}^{1}$. Los valores del GC e ITBV fueron indexados por superficie corporal (IC, ITBVI).

La medición del GC por TDP se efectuó con CAP 8,0 French (Abbott Thermodilution Catheter; Abbott Critical Care Systems; North Chicago, Illinois, USA) con inyección manual de $10 \mathrm{ml}$ de solución salina a temperatura ambiente no sincronizada con el ventilador. La PAOP se obtuvo al final de la espiración, sin desconectar el ventilador ni retirar la presión positiva de fin de espiración (PEEP). El monitoreo del GC por TDTP se efectuó con sistema PiCCO con inyección manual de 20 $\mathrm{ml}$ de solución salina fría $\left(<8^{\circ} \mathrm{C}\right)$.

Las mediciones se efectuaron en posición supina y la calibración del punto cero fue hecha a nivel de la línea axilar media. Los GC fueron medidos en triplicado, descartándose el valor cuya diferencia fuese mayor al $10 \%$.

\section{Sistema PICCO}

Este método requiere un catéter venoso central convencional al cual se conecta externamente un sensor capaz de medir la temperatura de la solución inyectada y un catéter arterial femoral 5.0 French (Pulsiocath PV2015L13; Pulsion Medical Systems; Munich, Alemania), que además de permitir la medición de la presión arterial, posee un sensor de temperatura en su extremo distal. 
El monitor PiCCO calcula el GC por análisis de la curva de TDTP usando la ecuación de StewartHamilton $^{11}$. A partir de la curva se obtiene el tiempo medio de tránsito (Mtt) y el tiempo de la pendiente de descenso exponencial (Dst) del indicador térmico. El producto del GC por el Mtt es igual al volumen térmico intratorácico total (ITTV) y el producto del GC por el Dst es equivalente al volumen térmico pulmonar total (PTV). La resta de ambos corresponde al volumen de fin de diástole global (GEDV) o volumen de las cuatro cámaras cardíacas ${ }^{9}$.

El ITBV se obtenía originalmente multiplicando el GC por el Mtt de un indicador colorimétrico de distribución intravascular (verde de indocianina). Dado que la única diferencia que existe entre el GEDV (indicador térmico) y el ITBV (indicador colorimétrico) es el volumen sanguíneo pulmonar (PBV) fue posible construir una ecuación de ajuste que permite calcular el ITBV a partir del GEDV, prescindiendo del indicador colorimétrico. El ITBV calculado a través del GEDV tiene buena correlación con el ITBV obtenido con indicador colorimétrico ${ }^{9,12}$.

Manejo ventilatorio. Los pacientes fueron ventilados en modo controlado por presión. El nivel de PEEP y la presión pico se ajustaron según el valor del punto de inflexión inferior y superior de la curva presión volumen obtenida con el método de flujo lento ${ }^{13}$. Cuando no fue posible identificar dichos puntos se utilizó la razón $\mathrm{PaO}_{2} / \mathrm{FiO}_{2}$ y una presión meseta $\leq 30 \mathrm{~cm}$ de $\mathrm{H}_{2} \mathrm{O}^{14}$.

Análisis de los datos. Se correlacionó el valor absoluto del GC obtenido por ambos métodos, utilizando el coeficiente de correlación de Pearson para asegurarnos que ambos eran equivalentes.

Con el fin de evaluar si una modificación del estimador de precarga tenía impacto sobre el GC se correlacionó la diferencia entre los valores de ITBVI $(\Delta$ ITBVI) y PAOP $(\Delta$ PAOP), con la correspondiente diferencia del IC del método respectivo ( $\Delta$ IC-PiCCO y $\Delta$ IC-CAP) entre dos mediciones consecutivas ( 6 am con 12 am y $6 \mathrm{pm}$ con $12 \mathrm{pm}$ ), utilizando el coeficiente de correlación de Pearson y se consideró un intervalo de confianza (IC) de 95\%.
Se utilizó t de Student para muestras pareadas para comparar $\Delta$ IC-CAP con $\Delta$ IC-PiCCO. El análisis estadístico se hizo con el programa SPSS versión 10.0 y los gráficos se construyeron con el programa Excel versión Windows XP ${ }^{\circledR}$. Se consideró estadísticamente significativo un valor de $\mathrm{p}$ $<0,05$. Este trabajo fue analizado por el Comité de Ética de Clínica Alemana, no existiendo objeciones éticas a su diseño y aplicación.

\section{RESULTADOS}

Se estudiaron 22 pacientes, 11 hombres y 11 mujeres. La media de la edad fue 60,8 \pm 19,4 años, del APACHE II y SOFA de ingreso $23,9 \pm 7$ y $10,3 \pm 3,2$ puntos, respectivamente. Todos presentaban inestabilidad circulatoria, 18 de origen séptico y 4 no séptico. El 68\% presentó síndrome de distress respiratorio agudo (SDRA) con una media del puntaje de injuria pulmonar (LIS) de $2,78 \pm 0,38$ puntos. La mortalidad global fue de $36 \%$. Tabla 1.

Se obtuvieron 343 perfiles hemodinámicos sucesivos por TDP (CAP) y TDTP (PiCCO), de modo que ambos métodos de monitoreo se encontraban bajo las mismas condiciones en relación a dosis de drogas vasoactivas y niveles de PEEP. La correlación entre los gastos cardíacos obtenidos por ambos métodos, realizada utilizando el total de las mediciones, fue estrecha ( $r=$ 0,87; $\mathrm{p}<0,01$; Figura 1).

Del total, 276 perfiles hemodinámicos correspondieron a los obtenidos a las $6 \mathrm{am}, 12 \mathrm{am}, 6 \mathrm{pm}$ y $12 \mathrm{pm}$. Los 67 restantes, destinados al manejo clínico de los enfermos, se efectuaron en horarios diferentes y fueron excluidos del segundo análisis. La correlación entre $\Delta$ ITBVI y $\Delta$ PAOP con el correspondiente $\Delta$ IC-PiCCO y $\Delta$ IC-CAP se realizó entre dos mediciones consecutivas, utilizando las 276 mediciones pareadas obtenidas en los horarios de protocolo, quedando un total de $138 \Delta$ para el análisis.

Se observó una correlación positiva significativa entre $\Delta$ ITBVI y $\Delta$ IC-PiCCO $(r=0,54$; IC $=0,41$ 0,65; $\mathrm{p}<0,01$; Figura 2). No hubo correlación entre $\Delta$ PAOP y $\Delta$ IC-CAP $(r=-0,097$; IC $=-0,07-0,26 ; \mathrm{p}=$ 0,19; Figura 3). Los $\Delta$ IC-CAP y $\Delta$ IC-PiCCO no fueron estadísticamente diferentes $(p=0,11)$. 
Tabla 1. C aracterísticas demográficas, ventilatorias y hemodinámicas de los pacientes durante el período de estudio

\begin{tabular}{|c|c|c|c|c|c|c|c|c|c|c|c|}
\hline Diagnósticos & Edad & APACHE II & LIS & NA max & NA min & DBT max & DBT min & SDRA & PEEP max & PEEP mim & Status \\
\hline SCPH & 42 & 11 & 2 & 0,03 & 0,01 & 3 & 1 & + & 14 & 10 & Vivo \\
\hline Peritonitis & 85 & 16 & - & 0 & 0 & 10 & 5 & - & 10 & 8 & Vivo \\
\hline NAC & 31 & 17 & 2,75 & 0,06 & 0,01 & 4 & 1 & + & 16 & 14 & Vivo \\
\hline Peritonitis & 51 & 18 & 3 & 0,08 & 0,01 & 5 & 2 & $+* * *$ & 18 & 8 & Vivo \\
\hline Peritonitis & 59 & 18 & 2,5 & 0,35 & 0,05 & 13 & 7,5 & $+* * *$ & 18 & 12 & Vivo \\
\hline Hemorragia alveolar & r 31 & 19 & 3,25 & 0,1 & 0,02 & 3 & 2 & + & 16 & 8 & Muerto \\
\hline PANH & 56 & 19 & - & 0,25 & 0,08 & 5 & 2 & - & 12 & 6 & Muerto \\
\hline NAC & 66 & 20 & 3 & 0,37 & 0,03 & 9 & 1 & + & 15 & 10 & Vivo \\
\hline NAC & 71 & 20 & - & 0,05 & 0,02 & 8 & 4 & - & 5 & 4 & Vivo \\
\hline Vasculitis & 42 & 22 & - & 0,04 & 0,01 & 12 & 3 & - & 15 & 10 & Muerto \\
\hline Mediastinitis & 73 & 23 & - & 0,4 & 0,01 & 8 & 8 & - & 16 & 10 & Vivo \\
\hline NAs & 21 & 24 & 2,5 & 0,04 & 0,02 & 2 & 1 & + & 18 & 10 & Vivo \\
\hline NAC & 82 & 24 & 3 & 0,18 & 0,08 & 6 & 1 & + & 8 & 5 & Muerto \\
\hline NAC & 75 & 24 & - & 0,12 & 0,03 & 5 & 1 & - & 10 & 8 & Vivo \\
\hline NAC & 80 & 26 & 3 & 0,15 & 0,01 & 10 & 2 & + & 16 & 6 & Vivo \\
\hline PANH & 75 & 27 & 2,5 & 0,42 & 0,02 & 14 & 10 & $+* * *$ & 16 & 13 & Muerto \\
\hline NAV & 45 & 27 & 3 & 0,15 & 0,1 & 6 & 5 & + & 10 & 10 & Muerto \\
\hline NAs & 49 & 30 & - & 0,11 & 0,01 & 3 & 1 & - & 14 & 9 & Vivo \\
\hline NAs & 91 & 33 & 2,5 & 0,35 & 0,03 & 5 & 2 & + & 14 & 10 & Vivo \\
\hline NAC & 76 & 34 & 2,75 & 0,25 & 0,01 & 15 & 3 & + & 16 & 8 & Vivo \\
\hline NAs & 66 & 37 & 3,5 & 0,23 & 0,01 & 8 & 2,5 & + & 22 & 13 & Muerto \\
\hline Sepsis cutánea & 71 & 37 & 2,5 & 1,2 & 0,2 & 6 & 5 & $+* * *$ & 15 & 8 & Muerto \\
\hline MEDIA ( \pm DS) & $60,8 \pm 19,4$ & $23,9 \pm 7,0$ & $2,78 \pm 0,38$ & $0,2 \pm 0,25$ & $0,04 \pm 0,04$ & $7,1 \pm 3,9$ & $3,32 \pm 2,6$ & $15 *$ & $14,3 \pm 3,8$ & $9,1 \pm 2,6$ & 8 ** \\
\hline
\end{tabular}

DBT: Dobutamina ( $\mu \mathrm{g} / \mathrm{Kg} / \mathrm{min}$.$) ; LS: Puntaje de injuria pulmonar; NA: Noradrenalina (\mu \mathrm{g} / \mathrm{Kg} / \mathrm{min}$.$) ; NAs:$ Neumonia Aspirativa; NAC: Neumonia Adquirida en la Comunidad; NAV: Neumonia Asociada a Ventilador; PANH: Pancreatitis Aguda Necro Hemorrágica; SDRA: Síndrome de Distress Respiratorio Agudo; SCPH: Síndrome Cardiopulmonar por Hanta Virus, PEEP: Presión positiva espiratoria final $\left(\mathrm{cm} \mathrm{H}_{2} \mathrm{O}\right)$ ${ }^{*}=$ Número de pacientes; ${ }^{* *}=$ Número de muertos; ${ }^{* * *}=$ SDRA extrapulmonar.

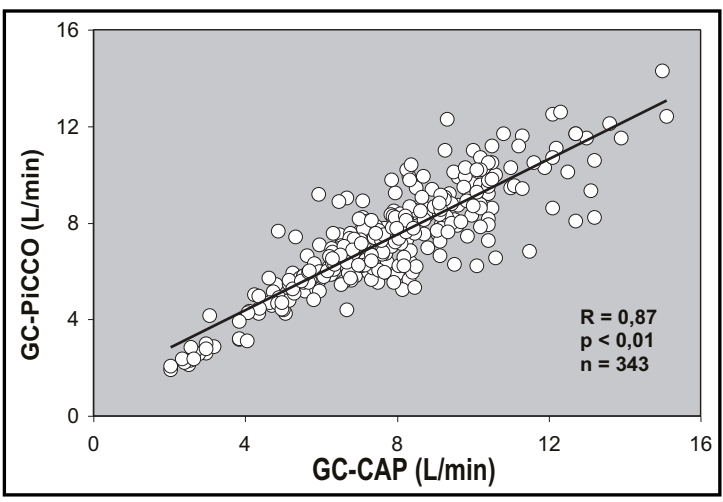

FigURA 1. Relación entre gasto cardíaco obtenido por TDP Y TDTP.

GC-CAP $=$ Gasto cardíaco catéter arteria pulmonar; GC-PiCCO = Gasto cardíaco sistema PiCCO.

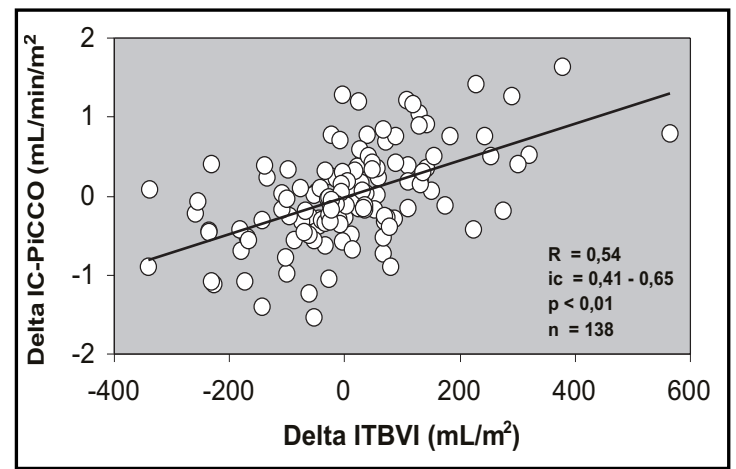

FIGURA 2. Correlación entre $\Delta$ ITBVI y $\Delta$ IC-PiCCO. $\Delta=$ delta. ic= Intervalo de confianza de $95 \%$. 


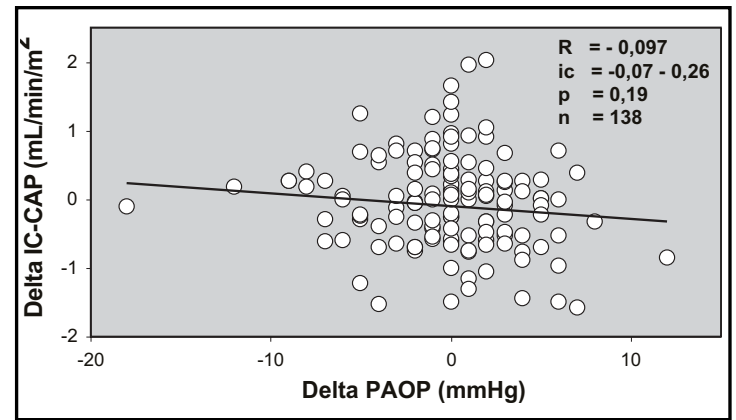

FIgURA 3. Correlación entre $\Delta$ PAOP y $\Delta$ IC-CAP. $\Delta=$ delta. ic= Intervalo de confianza de $95 \%$.

Los cambios positivos en el ITBVI $(n=72)$ fueron más a menudo asociados con aumentos del IC-PiCCO $(n=46 ; 63,9 \%)$ que cambios positivos en la PAOP $(n=58)$ con aumentos del IC-CAP $(n=25 ; 43,1 \%)$. Los cambios negativos en el ITBVI $(n=66)$ se acompañaron más frecuentemente de una disminución del IC-PiCCO $(n=46 ; 69,7 \%)$ que cambios negativos en la PAOP $(n=55)$ con disminución del IC-CAP ( $\mathrm{n}=28 ; 50,9 \%)$. Hubo 25 mediciones en que $\triangle$ PAOP fue cero, 14 se asociaron con un aumento del IC-CAP, 10 con una disminución y uno no se modificó.

\section{DisCUSIÓN}

Para evaluar la capacidad de las variables de precarga (PAOP e ITBVI) para predecir el IC nos basamos en la relación de dependencia que existe entre ambas variables. Antes de realizar el análisis corroboramos la presencia de una buena correlación tanto para el valor absoluto de GC como el $\Delta$ IC, hecho que ha sido confirmado previamente ${ }^{15-17}$. Fue relevante aclarar este aspecto debido a que el GC obtenido por TDP es muy sensible al incremento de la presión intratorácica, ya que ésta puede inducir una reducción del flujo sanguíneo en la arteria pulmonar e interferir por ende con la estimación del flujo sanguíneo sistémico $(\mathrm{GC})^{18}$. Este fenómeno no se observaría en la TDTP ya que el tiempo de circulación del indicador térmico, desde su inyección hasta que es detectado por el sensor femoral, incluye varios ciclos respiratorios ${ }^{19}$.

La precarga cardíaca corresponde a la tensión de la pared ventricular al final de la diástole y está condicionada por la longitud de la fibra muscular en reposo cuyo equivalente clínico es el volumen de fin de diástole del ventrículo izquierdo (VFDVI), parámetro no disponible en forma continua al lado de la cama de los pacientes. Asumiendo que la distensibilidad ventricular permanece constante los cambios en el volumen ventricular deberían ser reflejados por cambios recíprocos en la presión ventricular, por lo tanto, parámetros de presión tales como la presión de fin de diástole del VI, presión auricular izquierda y PAOP han sido utilizados en clínica como estimadores alternativos del VFDVI.

Al interpretar la PAOP debemos considerar esencialmente dos aspectos: la influencia de las variaciones de las dosis de vasopresores e inótropos sobre la post-carga y la contractilidad miocárdica y los efectos del incremento del volumen corriente y de la PEEP total (aplicada y auto-PEEP) sobre la presión intratorácica y la fosa pericárdi$\mathrm{ca}^{3,5-8}$. Ambos factores estuvieron presentes en nuestra población y pudieron contribuir a la mala correlación entre $\Delta$ PAOP y $\Delta$ IC-CAP.

$\mathrm{Si}$ bien el aumento de los volúmenes pulmonares puede influir en la medición de la PAOP, la PEEP cobra una importancia especial ya que permanecerá al final de la espiración, momento en que se realiza la medición. Además, la transmisión de la PEEP sobre la fosa pericárdica va a depender de la distensibilidad toracopulmonar, es decir, la transmisión será mayor si la reducción de la distensibilidad ocurre a expensas del componente torácico (ejemplo: SDRA extrapulmonar) y menor donde la reducción de la distensibilidad depende del componente pulmonar (SDRA pulmonar) ${ }^{8}$.

Del total de pacientes quince presentaron SDRA y, de ellos, $73 \%$ fue de origen pulmonar (neumonía); sin embargo, esta población no mostró mejor correlación entre $\Delta$ PAOP y $\Delta$ IC-CAP que aquellos con SDRA extrapulmonar.

Diversos estudios clínicos consideran que el ITBV sería un mejor indicador de precarga cardíaca por ser independiente de los cambios de la presión intratorácica y distensibilidad miocárdi$\mathrm{ca}^{8,20-24}$. En nuestros pacientes el $\Delta$ ITBVI tuvo correlación con $\Delta$ IC-PiCCO, tanto para cambios positivos como negativos del ITBVI, resultados que concuerdan con los hallazgos de LichtwarckAschoff y otros autores ${ }^{10,22,23}$. Si bien LichtwarckAschoff mostró un $\mathrm{r}=0,71$, él mantuvo sin 
variación las dosis de drogas vasoactivas entre cada medición. Sakka, modificó el soporte vasoactivo, pero sólo correlacionó los deltas entre las dos primeras mediciones y observó un $\mathrm{r}=0,67$, menor que el obtenido por el autor anterior. Nuestros datos, en cambio, muestran el comportamiento de las variables estudiadas a lo largo de todo el período de inestabilidad cardiopulmonar, situación que se acerca más a las condiciones que impone el manejo hemodinámico en las unidades de cuidados intensivos.

Aunque la variable de precarga volumétrica tuvo una mejor correlación que PAOP en predecir el IC nosotros esperamos observar una mejor correlación entre $\Delta$ ITBVI y $\Delta$ IC-PiCCO, sin embargo, hay que considerar que la precarga cardíaca no es el único determinante del GC ${ }^{7,11}$. En efecto, nuestros pacientes estuvieron sometidos a distintas dosis de drogas inotrópicas y vasopresoras

\section{REFERENCIAS}

1. Task Force of the American Colugge of Critical Care Medicine, Society of Critical Care Medicine. Practice parameters for hemodynamic support of sepsis in adult patients in sepsis. Crit Care Med 1999; 27: 639-60.

2. Goldenheim PD, Kazemi H. Current concepts. Cardiopulmonary monitoring of critically ill patients (Part 2). N Engl J Med 1984; 311: 776-80.

3. PINSKY MR. Clinical significance of pulmonary artery occlusion pressure. Intensive Care Med 2003; 29: 175-8.

4. Shippy C, Appel P, Shozmaker W. Reliability of clinical monitoring to assess blood volume in critically ill patients. Crit Care Med 1984; 12: 107-12.

5. Tomicic V, Andresen M, Romero C, Mercado M. Impacto hemodinámico de la presión positiva de fin de espiración (PEEP) durante la falla respiratoria grave: visión actual. Rev Méd Chile 2002; 130: 1419-30.

6. Teboul J, Pinsky M, Mercat A, Ángel N, Bernardin G, ACHARD J ET AL. Estimating cardiac filling pressure in mechanically ventilated patients with hyperinflation. Crit Care Med 2000; 28; 3631-6.

7. Sonnenblnk EH, Ross JR, Covel JN, Braunwald E. Alterations in resting length, tension relations of cardiac muscle induced by changes in contractile force. Circ Res 1966; 19: 980-8. que probablemente influyeron en las curvas de función ventricular a lo largo del tiempo.

En resumen, nuestros pacientes presentaron inestabilidad circulatoria grave, recibieron ventilación mecánica y soporte vasoactivo variable, condiciones que producen cambios frecuentes en la presión intratorácica, contractilidad miocárdica y post-carga ventricular izquierda. Sabemos que estos factores pueden ser una limitación importante al momento de estimar la precarga cardíaca a través de mediciones de presión, como queda demostrado con nuestros datos. Aunque la correlación de $\Delta$ ITBVI con el $\Delta$ IC-PiCCO en nuestro estudio fue inferior a la descrita por los autores citados, definitivamente PAOP no presentó ninguna correlación con el IC-CAP. Las ventajas atribuidas al monitoreo volumétrico hacen del ITBV una herramienta promisoria para optimizar el manejo hemodinámico en este tipo de pacientes.

8. Chapin JC, Downs JB, Douglas ME, Murphy EJ, Ruiz BC. Lung expansion, airway pressure transmission, and positive end expiratory pressure. Arch Surg 1979; 114: 1193-7.

9. SaKKa SG, RüHl CC, Pfeiffer UJ, Beale R, McLuckie A, ReINHART K ET AL. Assessment of cardiac preload and extravascular lung water by single transpulmonary thermodilution. Intensive Care Med 2000; 26: 180-7.

10. Lichtwarck-Aschoff M, Zeravik J, PFeiffer UJ. Intrathoracic blood volume accurately reflects circulatory volume status in critically ill patients with mechanical ventilation. Intensive Care Med 1992; 18: 142-7.

11. Leatherman J, Marini J. Clinical use of the pulmonary artery catheter. En: Hall J, ed. Principles of Critical Care. International Edition: Editorial McGraw-Hill, 1998; 155-76.

12. Tomicic V, Espinoza M, Hernández G, Abarca L. Monitoreo de la volemia y el agua pulmonar extravascular en UCI. Medicina Intensiva 2002; 17: 15-9.

13. Lu Q, Vieira S, Richecoeur J, Puybasset L, Kalfon P, CoRiat P ET AL. A simple automated method for measuring pressure-volume curves during mechanical ventilation. Am J Respir Crit Care Med 1999; 159: 275-82.

14. The Acute Respiratory Distress Syndrome Network. Ventilation with lower tidal volumes as compared with traditional tidal volumes for Acute Lung 
Injury and the Acute Respiratory Distress Syndrome. N Engl J Med 2000; 342: 1301-8.

15. SakKa S, Reinhart K, Meier-Helmann A. Comparison of pulmonary artery and arterial thermodilution cardiac output in critically ill patients. Intensive Care Med 1999; 25: 843-6.

16. PaVeK K, Lindquist O, Arfors K-E. Validity of thermodilution method for measurement of cardiac output in pulmonary oedema. Cardiovasc Res 1973; 7: 419-22.

17. Böck JC, Barker BC, Mackersie RC, Tranbaugh RF. Cardiac output measurement using femoral thermodilution in patients. J Crit Care 1989; 4: 106-11.

18. PINSKY MR. Determinants of pulmonary artery flow variations during respiration. J Appl Physiol 1984; 56: 1237-45.

19. Jansen JR, Schreuder JJ, Settels JJ, Kloek JJ, Vrsprile A. An adequate strategy for the thermodilution technique in patients during mechanical ventilation. Intensive Care Med 1990; 16: 422-5.

20. Buhre W, Weyland A, Schorn B. Changes in central venous and pulmonary capillary wedge pressure do not indicate changes in right and left heart volume in patients undergoing coronary artery bypass surgery. Eur J Anaesthesiol 1999; 16: 11-7.

21. Hoeft A, Schorn B, Weyland A, Scholz M, Buhre W, Stepanek E ET aL. Bedside assessment of intravascular volume status in patients undergoing coronary bypass surgery. Anesthesiology 1994; 81: 76-86.
22. Gödje O, Peyerl M, Seebauer T, Lamm P, Mair H, REICHART B. Central venous pressure, pulmonary capillary wedge pressure and intrathoracic blood volumes as preload indicators in cardiac surgery patients. Eur J Cardiothoracic Surg 1998; 13: 533-40.

23. Reuter DA, Felbinger TW, Moerstedt K, Weis F, SCHMidT C, KILger E et AL. Intrathoracic blood volume index measured by thermodilution for preload monitoring after cardiac surgery. J Cardiothorac Vasc Anesth 2002; 16: 191-5.

24. SakKa S, Bredle D, Reinhart K, Meier-Helumann A. Comparison between intrathoracic blood volume and cardiac filling pressures in the early phase of hemodynamic instability of patients with sepsis or septic shock. J Crit Care 1999; 14: 78-83.

Agradecimientos:

Al Sr. Luis Villarroel, Magíster en Estadística del Departamento de Salud Pública, Pontificia Universidad Católica de Chile, por su aporte en el procesamiento de los datos.

A todos los colegas residentes de nuestra unidad por su contribución desinteresada en el desarrollo del protocolo.

Conflictos de interés:

Los equipos PiCCO utilizados en el proyecto provienen de la empresa Gautier, Montevideo, Uruguay, sin representación en Chile. Declaramos no haber recibido ningún tipo de financiamiento desde la empresa Gautier ni Pulsion Medical Systems. 